\title{
Tremores Intra e Pós-Operatório: Prevenção e Tratamento Farmacológico*
}

\section{Intra- and Postoperative Tremors: Prevention and Pharmacological Treatment}

Viviane Ferreira Albergaria, TSA ${ }^{1}$, Michelle Nacur Lorentz, TSA², Frederico Augusto Soares de Lima ${ }^{3}$

\section{RESUMO}

Albergaria VF, Lorentz MN, Lima FAS - Tremores Intra e Pós-Operatório: Prevenção e Tratamento Farmacológico.

JUSTIFICATIVA E OBJETIVOS: Os tremores podem ocorrer como um efeito adverso da intervenção cirúrgica e anestesia. A incidência de tremores pós-operatórios varia entre 6,3\% e 66\%. Pacientes jovens, sexo masculino, uso de agentes anestésicos halogenados e tempo prolongado de anestesia ou procedimento cirúrgico estão relacionados com tremores. Os tremores são involuntários e apresentam-se como atividade muscular oscilatória com finalidade de aumentar a produção de calor. Tremor pós-operatório é uma desagradável complicação que está relacionada com o aumento de morbidade. O tremor aumenta o metabolismo, resultando em um acréscimo de $200 \%$ a $500 \%$ no consumo de oxigênio.

CONTEÚDO: Discute as causas, a prevenção e o tratamento dos tremores intra- e pós-operatório em pacientes adultos e pediátricos submetidos à intervenção cirúrgica sob anestesia geral ou do neuroeixo.

CONCLUSÕES: Os tremores são, juntamente com náuseas e vômitos, causas de intenso desconforto na sala de recuperação pósanestésica, além de potencialmente prejudiciais por gerarem aumento da demanda metabólica. Embora a presença de tremores não tenha sido diretamente relacionada com a morbidade cardíaca, a prevenção tem se tornado tema de debate e de vários artigos científicos. A prevenção e o tratamento de tremores devem ser implementados. Pacientes com reserva cardiopulmorar limitada podem sofrer com acidose láctica, dessaturação venosa mista e hipoxemia.

Unitermos: ANESTESIA: Geral, Locorregional; COMPLICAÇÕES: hipotermia, tremor; TREMOR: prevenção, tratamento.

${ }^{\star}$ Recebido do (Received from) Instituto Biocor, Nova Lima, MG

1. Anestesiologista do Corpo Clínico do Instituto Biocor; Anestesiologista e Co-Responsável pelo CET do Hospital das Clínicas da UFMG

2. Anestesiologista do Corpo Clínico do Instituto Biocor

3. $\mathrm{ME}_{3}$ da Fundação Hospitalar do Estado de Minas Gerais (FHEMIG)

Apresentado (Submitted) em 19 de maio de 2006

Aceito (Accepted) para publicação em 23 de março de 2007

Endereço para correspondência (Correspondence to):

Viviane Ferreira Albergaria

Praça Clemente de Faria, 80 - Prado

30410-490 Belo Horizonte, MG

E-mail: vivialbergaria@yahoo.com.br

(C) Sociedade Brasileira de Anestesiologia, 2007

\section{SUMMARY}

Albergaria VF, Lorentz MN, Lima FAS - Intra- and Postoperative Tremors: Prevention and Pharmacological Treatment.

BACKGROUND AND OBJECTIVES: Tremors can be an adverse effect of the surgical intervention and anesthesia. The incidence of postoperative tremors varies from $6.3 \%$ to $66 \%$. Young age, male gender, the use of halogenated anesthetics, and prolonged anesthesia or surgical procedure are related with tremors. Tremors are involuntary and present as oscillating muscular activity aiming at increasing heat production. Postoperative tremors are a disagreeable complication related with increased morbidity. Tremors increase the metabolism, resulting in a $200 \%$ to $500 \%$ increase in oxygen consumption.

CONTENTS: The objective of this paper was to discuss the causes, prevention, and treatment of intra- and postoperative tremors in adults and children undergoing general anesthesia or neuroaxis anesthesia for surgical interventions.

CONCLUSIONS: Tremors, along with nausea and vomiting, cause severe discomfort in the recovery room, besides being highly prejudicial because they generate an increase in the metabolism. Although the presence of tremors has not been directly related with cardiac morbidity, its prevention has become the subject of discussion and several scientific reports. Prevention and treatment of tremors should be implemented. Patients with limited cardiovascular reserve could develop lactic acidosis, mixed venous desaturation, and hypoxemia.

Key Words: ANESTHESIA: General, regional; COMPLICATIONS: hypothermia, tremor; TREMOR: prevention, treatment.

\section{INTRODUÇÃO}

Em espécies homeotérmicas, o sistema de regulação térmica coordena defesas contra o frio e o calor e mantém a temperatura corporal interna dentro de uma estreita variação ideal para as funções fisiológicas e metabólicas. Entretanto, a combinação de anestesia e exposição ao frio pode causar hipotermia e tremores nos pacientes cirúrgicos ${ }^{1,2}$. Os tremores quase sempre são autolimitados, não se tornam crônicos e, em geral, não geram conseqüências mais sérias, por isso na maioria das vezes são subestimados.

É comum o tremor pós-operatório estar associado à perda de calor corporal, embora a hipotermia por si só não explique completamente a sua ocorrência. Entretanto, ele afeta o conforto dos pacientes e, algumas vezes, pode levar a complicações ${ }^{3}$. 
Junto com náusea e vômito, o tremor é um dos problemas mais freqüentes na fase de recuperação inicial após anestesia geral ${ }^{4,5}$. O tremor é muito incômodo e psicologicamente estressante. Pode também gerar complicações, sobretudo em pacientes com doença coronariana devido ao aumento do consumo de oxigênio $\left(\mathrm{O}_{2}\right)$, de $100 \%$ a $600 \%$; produção de gás carbônico $\left(\mathrm{CO}_{2}\right)$, resultando em maior ventilação por minuto e aumento no débito cardíaco ${ }^{6}$; circulação de catecolaminas (aumento da freqüência cardíaca); além de diminuir a saturação de $\mathrm{O}_{2}$ no sangue venoso misto ${ }^{7-9}$. É possível, também, haver aumento da pressão intracraniana e intra-ocular, interferência com ECG, aferição da oximetria de pulso ${ }^{10}$, pressão arterial, aumento do metabolismo e acidose láctica ${ }^{7-9}$.

O tremor pós-operatório pode ser de dois tipos. O primeiro corresponde ao tremor relativo à regulação térmica que está associada à vasoconstrição, sendo uma resposta fisiológica à hipotermia central ou periférica ${ }^{11,12}$ desenvolvida no período intra-operatório. O segundo corresponde ao tremor associado à vasodilatação ou tremor não relacionado com a regulação térmica ${ }^{13}$. Observou-se que tremores não ocorrem apenas em pacientes hipotérmicos ${ }^{14,15}$, eles podem também acometer pacientes normotérmicos ${ }^{2,16,17} \mathrm{e}$, em contraposição, muitos pacientes com hipotermia importante não apresentam tremores. Sessler e col. concluíram que o tremor pós-operatório tem origem na regulação térmica ${ }^{12}$, é provocado por hipotermia e pode também ocorrer em indivíduos normotérmicos que estão desenvolvendo febre ${ }^{15}$. Porém, alguns tremores durante o parto ${ }^{18}$ e após anestesia geral ${ }^{19}$ não são relacionados com a regulação térmica e são agravados pela dor mal controlada ${ }^{8,20}$.

Há mais de dez anos, Crossley ${ }^{21}$ determinou algumas variáveis que influenciam o desenvolvimento de tremores: intervenções cirúrgicas mais longas, sexo masculino, anticolinérgicos (atropina), ventilação espontânea, estado físico alterado (ASA elevado), procedimento cirúrgico geral comparado com o ortopédico e a administração de sangue. Ele também relatou que a idade avançada e a administração de propofol, alfentanil ou morfina foram os fatores protetores mais importantes contra tremores.

\section{FISIOPATOLOGIA}

Muitos estudos sugerem que os fatores relacionados com a intervenção cirúrgica, como dor e estresse, contribuem para a gênese do tremor ${ }^{12}$. São exemplos os mecanismos envolvidos no aparecimento de tremores e, presumivelmente, são resultado de adaptação evolutiva. Não existe via única responsável por tremores; ao contrário, vários são os mecanismos capazes de modular as respostas de regulação térmica 15 .

A hipotermia intra-operatória, além de causar graves complicações, pode prolongar o tempo de internação ${ }^{22}$ e estar também associada a diminuição do metabolismo de fár- macos, atraso no despertar anestésico, balanço nitrogenado negativo e tremores ${ }^{23}$.

O uso de hipotermia como estratégia terapêutica para neuroproteção permanece controvertido ${ }^{24}$. A hipotermia reduz a pressão intracraniana, aumenta a pressão de perfusão encefálica e diminui o seu metabolismo. Entretanto, mesmo a hipotermia moderada apresenta efeitos colaterais indesejáveis, como tremores e vasoconstrição periférica, resultando em instabilidade hemodinâmica, aumento do consumo de oxigênio e isquemia encefálica e miocárdica.

Em seres humanos, a hipotermia moderada resulta em meIhor recuperação neurológica em pacientes sobreviventes à parada cardiorrespiratória ${ }^{25,26}$. O International Liaison Committee on Resuscitation (ILCOR) recomenda seu uso nesses casos ${ }^{27}$.

O tremor pós-operatório ocorre em $5 \%$ a $65 \%$ dos pacientes em recuperação de anestesia geral ${ }^{7}$ e em $30 \%$ dos submetidos à anestesia peridural ${ }^{28}$. Podem ser tremores relacionados com a regulação térmica ou com a liberação de citocinas pelo procedimento cirúrgico. Em geral, a temperatura central diminui de $0,5^{\circ}$ a $1,5^{\circ} \mathrm{C}$ na primeira hora após a indução anestésica. A hipotermia que se desenvolve na primeira hora após a indução da anestesia geral ${ }^{29}$ ou neuroaxial ${ }^{30}$ resulta primariamente de redistribuição do calor do centro para a periferia. Todos os anestésicos, opióides e sedativos diminuem a vasoconstrição e o controle autonômico de regulação térmica (limiar de tremor) facilitando a hipotermia.

Em um estudo com 2.595 pacientes adultos, Crossley ${ }^{21}$ detectou tremores em 164 (6,5\%), e identificou como fatores de risco o sexo masculino e a medicação pré-anestésica realizada com anticolinérgicos. O tipo de indução anestésica não foi considerado fator de risco nesse estudo. Outro estudo, realizado por Eberhart e col. ${ }^{31}$, avaliou 1.340 pacientes consecutivos submetidos à intervenção cirúrgica sob anestesia geral e observou tremores em 11,9\%. Os fatores de risco relacionados com tremores pós-operatórios foram hipotermia, idade, procedimentos cirúrgicos ortopédicos e intervenções cirúrgicas prolongadas; e a idade pareceu ser o fator mais importante. Teve como objetivo desenvolver algoritmo para prever tremores, foram estudados pacientes submetidos à intervenção cirúrgica, excluindo os que apresentavam hipertermia pré-operatória ou os que usavam medicações que pudessem influenciar a regulação térmica (clonidina, meperidina, fenotiazina). A incidência de tremor moderado ou intenso (graus 3 e 4 ) foi de 11,6\%. A incidência total de qualquer tremor (incluindo grau 2) foi de $14,4 \%$. Atividade de regulação térmica (incluindo vasoconstrição e piloereção) foi observada em 17,6\%. Entre esses pacientes apenas $33 \%$ reclamaram de frio. O trabalho apresentado utilizou análise multivariável e encontrou três preditores independentes de risco: juventude, procedimento cirúrgico com endoprótese e temperatura corporal central baixa. A idade foi, de longe, o fator de risco mais importante para o 
tremor, sendo responsável por mais de $70 \%$ do poder preditivo de todo o modelo. Isso não foi surpresa porque a resposta de regulação térmica é diminuída em idosos ${ }^{15}$.

É difícil determinar fatores de risco independentes para tremores antes da intervenção cirúrgica, já que numerosas variáveis influenciam a regulação térmica no pós-operatório resultante dos anestésicos voláteis ${ }^{32}$ ou venosos e dos sedativos ${ }^{33}$. Além disso, quase todos os fármacos usados regularmente pelos pacientes (anti-hipertensivos e antidiabéticos, derivados cumarínicos) parecem oferecer proteção significativa para tremores. De fato há uma incidência bem menor de tremor em pacientes em uso de propranolol ${ }^{34}$.

Tremor e vasoconstrição são controlados em $30 \%$ pela temperatura central e em $20 \%$ pela temperatura média da pele ${ }^{35}$. É, portanto, razoável presumir que a hipotermia contribui para tremor e que a normotermia é protetora, na ausência de intervenção cirúrgica ${ }^{12}$. O estudo demonstrou que a temperatura central teve apenas uma leve influência no desenvolvimento de tremores comparada com a idade (fator mais determinante) e foi compatível com resultados de outros estudos que mostraram que a temperatura tem um fraco poder preditivo para tremor ${ }^{36,37}$. De acordo com a análise de regressão logística multifatorial a temperatura central não foi diretamente relacionada com tremores pósoperatórios em crianças, enquanto a mudança relativa de temperatura perioperatória foi um dos três preditores independentes ${ }^{38}$. Porém, pacientes com temperaturas corporais menores que $36^{\circ} \mathrm{C}$ sofreram tremores por mais tempo do que aqueles que foram aquecidos ${ }^{39}$.

O trabalho confirmou o resultado anterior ${ }^{21}$ de que o procedimento cirúrgico ortopédico (particularmente endoprótese) usando cimento ósseo foi um fator de risco independente para tremor. Uma explicação possível para isso seria a liberação de citocinas (como fator de necrose tissular-alfa e interleucina-6) estimulada pelo cimento ósseo polimetilmetacrilato ${ }^{40}$.

A intervenção cirúrgica de longa duração foi associada à maior freqüência de tremor. Uma explicação possível é que esses procedimentos são mais complexos e invasivos. $O$ tecido lesado pode liberar substâncias pirogênicas que modificam o ponto de gatilho do sistema de regulação térmica no pós-operatório, induzindo os tremores ${ }^{41}$.

O aquecimento ativo no intra-operatório para manter normotermia é importante para a prevenção de tremores ${ }^{42,43}$. O centro de regulação térmica está localizado no hipotálamo posterior e sua atividade é modulada pelos receptores de temperatura na pele, nas vísceras e em vários níveis do neuroeixo ${ }^{42,44}$. Todos os anestésicos voláteis mostraram produzir tremor pela alteração do limiar de tremor ${ }^{45,46}$.

Durante a anestesia o paciente não apresenta respostas de regulação térmica porque o reflexo está inibido. Durante a recuperação da anestesia a inibição desaparece e os tremores se iniciam quando a temperatura está abaixo do limiar de regulação térmica ${ }^{47}$.

\section{TREMORES E ANESTESIA}

Como a hipotermia é fator preditivo de tremores, constituindo um fator independente de risco de eventos mórbidos cardíacos ${ }^{48}$, ela deve ser evitada, reconhecida e tratada. Embora no intra-operatório ela possa ser benéfica, pois diminui o metabolismo e protege órgãos vitais de isquemia, quando o paciente emerge da anestesia as respostas adrenérgicas e metabólicas de regulação térmica são ativadas causando desconforto pós-operatório, tremores, aumento de sangramento, aumento da incidência de infecção e efeitos cardiovasculares ${ }^{49}$. No período intra-operatório as incidências de isquemia do miocárdio e taquicardia ventricular são similares nos pacientes hipotérmicos e nos normotérmicos; já no pós-operatório a incidência de taquicardia ventricular e de alterações eletrocardiográficas é maior nos pacientes hipotérmicos ${ }^{48}$. A disparidade entre o período intra- e o pós-operatório sugere que a anestesia pode ter efeito protetor nas respostas adversas ao estresse causado pelo frio. Existem dois mecanismos de respostas de regulação térmica a hipotermia corporal que podem ser deletérios para o sistema cardiovascular: resposta adrenérgica com vasoconstrição acompanhada de elevação da pressão arterial e aumento da

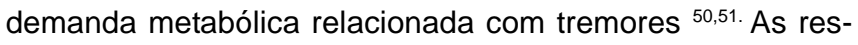
postas hemodinâmicas à vasoconstrição decorrente da regulação térmica parecem ser similares sem anestesia e com diferentes concentrações de desflurano e isoflurano ${ }^{52}$

\section{GRADUAÇÃO DOS TREMORES}

O grau dos tremores foi avaliado e classificado de acordo com uma escala qualitativa no artigo de Crossley e Mahajan ${ }^{36}: 0=$ sem tremor; 1 = sem atividade muscular visível, mas com piloereção, vasoconstrição periférica ou ambos; 2 = atividade muscular em apenas um grupo muscular; 3 = atividade muscular moderada em mais de um grupo muscular mas sem tremor generalizado; e 4 = atividade muscular violenta envolvendo todo o corpo.

\section{PREVENÇÃO FARMACOLÓGICA DE TREMORES}

O aquecimento adequado do corpo nem sempre é possível. Deve-se, portanto, em pacientes selecionados, prevenir os tremores utilizando técnicas farmacológicas.

Várias medicações já foram estudadas como profilaxia e tratamento incluindo clonidina, meperidina, cetanserina (fármaco anti-hipertensivo, antagonista com alta afinidade para receptores 5-HT e receptores adrenérgicos $\alpha_{1}$ ); doxapram (estimulante respiratório); tramadol e outros opióides ${ }^{15,53}$. O sistema colinérgico provavelmente está entre os mediadores do tremor. Fisostigmina, um anticolinesterásico que cruza a barreira hematoencefálica, é amplamente utilizada para tratar a síndrome anticolinérgica central ${ }^{54}$. Estudos sugerem que a fisostigmina aumenta a neurotransmissão colinér- 
gica, via estímulo do eixo hipotálamo-hipófise-supra-renal e medula adrenal, levando à inibição da regulação térmica generalizada.

A hipotermia moderada pode apresentar efeitos indesejáveis como tremores e vasoconstrição e medidas para prevenilos no pós-operatório podem ser necessárias. Existem relatos que a clonidina reduz a incidência de tremores no pós-operatório, porém o mecanismo de ação não está claro ${ }^{54}$. Agonistas $\alpha_{2}$ agem em receptores adrenérgicos $\alpha_{2} \mathrm{e}$ imidazólicos. Os primeiros estão localizados no encéfalo, namedula espinhal e na periferia. No encéfalo eles relacionam-se com efeito sedativo; na medula espinhal, com analgesia. A ação da clonidina nos receptores $\alpha_{2}$ pós-sinápticos no sistema nervoso central reduz o tônus simpático que leva à vasodilatação periférica e hipotensão arterial ${ }^{24}$. Os efeitos da meperidina na regulação térmica parecem envolver receptores adrenérgicos $\alpha_{2}{ }^{41}$.

Vários fármacos (meperidina, cetanserina, sufentanil, alfentanil, tramadol, fisostigmina, urapidil, nefopam, doxapram e nalbufina) já foram testados para prevenir ou tratar tremores ${ }^{7-9,28,55,56}$. A meperidina mostrou ser um dos mais efetivos ${ }^{56,57}$, agindo provavelmente no centro de regulação térmica ${ }^{55}$ ou via receptores opióides ${ }^{15}$. É provável que o receptor NMDA também module a regulação térmica em vários níveis ${ }^{15}$. A cetamina, um antagonista NMDA, mostrou ser capaz de inibir tremores ${ }^{58}$.

Os tratamentos estabelecidos para prevenir tremores, incluem vários fármacos como nefopam, uma benzoxazocina que inibe a recaptação de noradrenalina, dopamina e serotonina. Esses fármacos não são específicos contra tremores e exercem seus efeitos por meio de mecanismos diferentes como diminuição da ativação do sistema nervoso simpático ${ }^{9,53,59}$, supressão adrenal ${ }^{38}$, estimulação de receptores opióides ou influenciando no sistema serotoninérgico $5,9,15,60$.

O sistema colinérgico é uma via importante que potencialmente influencia no tremor. O hipotálamo e o corpo estriado são as regiões encefálicas nas quais a fisostigmina tem maior atividade ${ }^{61,62}$. A fisostigmina é um inibidor da colinesterase de ação central e interage com receptores encefálicos colinérgicos muscarínicos, serotoninérgicos, endorfinérgicos, assim como interações com agonistas opióides- $\mu$ e $\alpha_{2}$-agonistas ${ }^{15}$. Portanto, seus mecanismos são mais por aumento da neurotransmissão central. Ela parece ser segura e altamente efetiva com mínimos efeitos colaterais que incluem alterações hemodinâmicas; porém eles não foram observados no estudo, provavelmente pela administração lenta (15 minutos) ${ }^{62,63}$.

Além disso, a fisostigmina produziu analgesia pela liberação de $\beta$-endorfinas ${ }^{64}$; e dor pós-operatória pode levar à presença de tremores ${ }^{20}$. Em geral, a dose usada de fisostigmina é de $2 \mathrm{mg}{ }^{62,63}$. A fisostigmina reduziu significativamente a incidência e a gravidade dos tremores tanto quando o nefopam. As vias colinérgicas parecem estar envolvidas na regulação térmica, levando a diminuição do tremor sem alterar o despertar da anestesia, a vigília e a recuperação no pós-operatório.

Em outro estudo, dividido em duas partes, Stapelfeldt e col. ${ }^{24}$ investigaram o uso da clonidina em 48 pacientes submetidos à neurocirurgia eletiva. Primeiro determinaram, em 14 pacientes, a dose de clonidina necessária para prevenir tremores no pós-operatório após indução de hipotermia moderada $\left(35^{\circ} \mathrm{C}\right)$. Essa dose foi de $3 \mu \mathrm{g} \cdot \mathrm{kg}^{-1}$ A outra parte do estudo pesquisou se a clonidina retardaria o despertar da anestesia em neurocirurgia, em vista de seu efeito sedativo, atrasando a avaliação neurológica. Trinta e quatro pacientes submetidos a procedimento neurológico foram distribuídos em dois grupos de forma aleatória para receberem clonidina $\left(3 \mu \mathrm{g} \cdot \mathrm{kg}^{-1}\right)$ ou solução fisiológica a $0,9 \%$ assim que se iniciasse o fechamento da dura-máter. Os anestésicos inalatórios e a infusão de opióides eram interrompidos nesse momento e, em todos os pacientes, iniciava-se infusão de propofol. A recuperação do paciente era avaliada duas horas após o término da anestesia nos seguintes momentos: ao abrir os olhos, na extubação, quanto à obediência ao comando e orientação em relação ao tempo e espaço. Não houve diferença significativa entre os dois grupos ao considerar o tempo de recuperação pós-anestésica. Porém, a pressão arterial sistólica foi menor no grupo que recebeu clonidina quando comparado com o grupo-controle. Esses valores apresentaram diferença estatística significativa, porém, sem importância clínica evidente ${ }^{24}$. A dose ideal de clonidina ainda não está bem definida, mas pequenas doses podem ser suficientes para prevenir o tremor após hipotermia moderada.

Piper e col. ${ }^{9}$ compararam a clonidina com o nefopam para prevenção de tremores e encontraram eficácia semelhante, porém houve maior sedação com clonidina além de prolongamento no despertar, sugerindo que o nefopam é o melhor fármaco profilático.

Em outra revisão sistematizada, Kranke e col. ${ }^{65}$ descreveram a eficiência e os riscos da prevenção de tremores utilizando fármacos. Foram analisados 27 artigos (1.348 adultos receberam medicação e 931 eram controles). A incidência de tremores nos controles foi extremamente freqüente (52\%). Clonidina 65 a $300 \mu \mathrm{g}$ (1.078 pacientes), meperidina 12,5 a $35 \mathrm{mg}$ (250 pacientes), tramadol 35 a $220 \mathrm{mg}$ (250 pacientes) e nefopam 6,5 a $11 \mathrm{mg}$ (204 pacientes) foram testados em pelo menos três estudos cada. Todos foram mais efetivos comparados com os controles. Os artigos sugerem que menos de quatro pacientes têm de receber clonidina profilática para que um não tenha tremores após o procedimento cirúrgico. Para meperidina, nefopam e tramadol a eficiência pareceu ser ainda mais convincente. A alta prevalência de tremores nos pacientes não-tratados sugere que as populações dos estudos têm um risco muito alto, o que pode não representar a população geral, além de que a alta prevalência nessas populações tende a superestimar a utilidade das intervenções terapêuticas. A clonidina foi testada em mais 
estudos que os outros fármacos, o que permitiu avaliar o efeito de sua dose e o tempo de administração. Para maior eficiência ela deve ser administrada na indução (e não antes) em doses superiores a $140 \mu \mathrm{g}{ }^{65}$.

Houve fatores limitantes. Primeiro, nenhum dos fármacos analisados tem seu mecanismo de ação, contra tremores, totalmente elucidado. Alguns podem alterar o limiar de tremor. Isso foi demonstrado para meperidina ${ }^{66}$ e clonidina ${ }^{67}$. Segundo, não foi possível estabelecer fatores preditivos para tremores pós-operatórios. Terceiro, como num estudo similar prévio ${ }^{41}$, foi preciso utilizar comparações indiretas para estimar a eficiência relativa das intervenções antitremores já que não há uma intervenção padrão-ouro que possa ser usada como comparação. Quarto, a maioria dos trabalhos tem tamanho de amostra pequeno, apenas cinco estudos tinham 50 pacientes ou mais ${ }^{68,71}$, e estudos com amostras pequenas tenderam a superestimar a eficácia dos tratamentos. Quinto, a qualidade do método da maioria dos estudos foi insatisfatória. E sexto, houve um relato pobre de efeitos adversos, o que não significa que eles não ocorreram. Dal e col. ${ }^{72}$ compararam baixas doses de cetamina, meperidina e placebo para prevenir tremores. Foram estudados 90 pacientes com idade entre 18 e 65 anos, estado físico ASA I ou II, submetidos à anestesia geral por 60 a 180 minutos, divididos em três grupos de 30 . O Grupo $S$ recebeu solução fisiológica a 0,9\%, o Grupo K recebeu cetamina $\left(0,5 \mathrm{mg} \cdot \mathrm{kg}^{-1}\right)$ e o Grupo P recebeu meperidina $(20 \mathrm{mg}) 20$ minutos antes da intervenção cirúrgica. A temperatura timpânica de todos manteve-se superior a $36^{\circ} \mathrm{C}$ e, portanto, não exigiram aquecimento. Os grupos $\mathrm{K}$ e $\mathrm{P}$ tiveram número significativamente menor de tremor do que o grupo $S$ e não houve diferença entre os grupos K e P. O tempo para solicitação de analgésicos no grupo $S$ foi bem menor (12 min) que nos grupos $\mathrm{K}$ (25 min) e $\mathrm{P}$ (32 min). Duas horas após o término do procedimento cirúrgico todos os pacientes necessitaram de analgésicos. Não houve diferença nos escores de dor entre os grupos utilizando a Escala Visual Análoga (VAS) 30 min após o término da operação. Dois pacientes do Grupo K tiveram tremores grau 2 e foram tratados com meperidina que foi efetiva, provavelmente porque ela tem mecanismo de ação diferente ou porque a dose de $0,5 \mathrm{mg} \cdot \mathrm{kg}^{-1}$ de cetamina não foi suficiente. Nenhum paciente apresentou efeitos adversos atribuíveis à cetamina. A cetamina pode ser uma alternativa para profilaxia de tremores pós-operatórios em pacientes que não podem receber meperidina. Estudos futuros devem mostrar a dose ideal de cetamina para esse fim. Não houve diferença entre os grupos $\mathrm{K}$ e $\mathrm{P}$ na prevenção de tremores. Não houve relação entre a temperatura axilar e 0 tremor ${ }^{58}$.

A cetamina é um antagonista NMDA que deve agir contra tremores via termogênese sem tremores, ação no hipotálamo ou efeito $\beta$-adrenérgico, além de ter algum efeito no receptor $\kappa$. Um estudo sugere que a meperidina deve agir contra tremor via receptores $\kappa$, pois altas doses de naloxona inibem sua ação (inibem receptores mu e kapa) enquanto pequenas doses de naloxona não o fazem (inibem apenas receptores $\mathrm{mu})^{56}$. A desvantagem da meperidina é o risco de depressão respiratória, náusea e vômito.

Rohm e col. ${ }^{73}$ pesquisaram os efeitos da fisostigmina na prevenção de tremores pós-operatórios, comparando-a com 0 nefopam, um agente sabidamente efetivo ${ }^{5}$. Foram estudados 89 pacientes submetidos a procedimento cirúrgico abdominal ou urológico. Os pacientes receberam fisostigmina $2 \mathrm{mg}(\mathrm{n}=31)$, nefopam $10 \mathrm{mg}(\mathrm{n}=30)$ ou solução fisiológica a $0,9 \%(n=28)$. Os parâmetros hemodinâmicos e a temperatura foram medidos na indução e 5, 15, 30 e 60 minutos após chegada na sala de recuperação pós-anestésica. Houve muito menos tremor após a administração de fisostigmina e nefopam $(9,7 \%$ e $3,3 \%)$ em comparação com o placebo (53,6\%). Tempo de extubação, parâmetros hemodinâmicos e temperatura timpânica foram bem similares em todos os grupos. Apenas o nefopam reduziu de forma significativa as náuseas e os vômitos pós-operatórios. A fisostigmina reduziu com sucesso e segurança a incidência e a gravidade dos tremores. Esse sucesso foi similar ao do nefopam e não prolongou o tempo de recuperação do paciente.

Outro estudo realizado por Hong e Lee ${ }^{74}$ em pacientes submetidas à cesariana com raquianestesia que foi realizada com 8 a $10 \mathrm{mg}$ de bupivacaína hiperbárica a 0,5\%, associada à morfina $(0,1 \mathrm{mg}$ ou $0,2 \mathrm{mg})$, à meperidina $(10 \mathrm{mg})$ ou apenas ao anestésico local (AL). As pacientes que receberam apenas $A L$ apresentaram maior incidência de tremores $(23,3 \%)$. Já as que receberam meperidina apresentaram, além de menor incidência (3,3\%), menor intensidade de tremores, mesmo quando comparadas com as que receberam morfina $(0,1 \mathrm{mg}-17 \% ; 0,2 \mathrm{mg}-13,3 \%)$. A administração de opióide, por via peridural, também pode apresentar a propriedade de abolir os tremores, embora os mecanismos de ação desses opióides, influenciando no limiar do tremor, ainda não estejam bem estabelecidos ${ }^{75}$. Abreu e col. ${ }^{75}$ realizaram um estudo com o objetivo de comparar o bloqueio peridural com e sem fentanil, quanto à incidência de tremores no intra e pós-operatório. Concluiu-se que a adição de $100 \mu \mathrm{g}$ de fentanil ao AL, por via peridural, não tem a propriedade de abolir o tremor, mas de reduzir a sua incidência e a intensidade, sem aumentar a incidência de náuseas e vômitos.

\section{PREVENÇÃO NÃO-FARMACOLÓGICA DE TREMORES}

O tremor pode ser tratado com aquecimento, aplicação de calor radiante ou fármacos ${ }^{55}$. Dentre as medidas utilizadas para manutenção da temperatura corporal o uso do cobertor com ar aquecido parece ser eficiente e de custo adequado ${ }^{43}$. Simplesmente cobrir o paciente com um cobertor foi indicado como redutor de tremor sem alterar a temperatura central ${ }^{43}$. A temperatura da pele não é um fator preditivo de tremor. Porém, é inquestionável que o aumento suficiente da temperatura da pele pode, sozinho, interromper o tremor. 


\section{TRATAMENTO DOS TREMORES PÓS-OPERATÓRIOS}

O tremor é uma complicação freqüente na sala de recuperação pós-anestésica (SRPA) e existem vários medicamentos utilizados para o seu tratamento, embora seus mecanismos de ação não estejam totalmente esclarecidos ${ }^{41}$; em metanálise realizada por Kranke e col. a meperidina aparece como o fármaco mais efetivo para o tratamento de tremores e na dose de $25 \mathrm{mg}$ reduziu a recorrência de tremores nove vezes mais que o placebo, mantendo um efeito antitremor por pelo menos 45 minutos. A meperidina foi associada a pequeno aumento de efeitos colaterais como náuseas, vômitos e diminuição da freqüência respiratória. Nas situações em que a meperidina não pode ser usada os autores sugerem o uso de clonidina $(150 \mu \mathrm{g})$, cetanserina (10 mg), alfentanil $(0,25 \mathrm{mg}$ ) ou doxapram (100 mg). Deve ser lembrado que a manutenção da temperatura corporal intra-operatória e o uso de cobertores com ar aquecido no intra- e pós-operatório diminuem a necessidade de medicamentos para tratamento de tremores ${ }^{76}$.

Em estudo realizado em 220 pacientes com o objetivo de identificar a dose eficaz de meperidina para o tratamento de tremores, Crossley ${ }^{76}$ concluiu que $0,35 \mathrm{mg} \cdot \mathrm{kg}^{-1}$ é a menor quantidade necessária para tratar tremor pós-operatório de forma satisfatória.

O uso do tramadol para tratamento de tremores tem sido feito nos últimos anos, e a sua eficácia é semelhante à da meperidina segundo alguns estudos ${ }^{77}$.

O nefopam é uma benzoxacina analgésica de ação central que reduz os tremores sem comprometer a ventilação, com mínimos efeitos sedativos e com eficiência comprovada. Ao contrário dos outros fármacos, não atua como vasoconstritor, apenas na via do tremor ${ }^{78}$.

\section{TREMORES EM PACIENTES PEDIÁTRICOS}

A maior parte dos estudos envolvendo tremor pós-operatório é realizada em adultos e, quando em crianças, inclui um número pequeno de pacientes ${ }^{16}$. Em um clássico artigo, Akin e col. ${ }^{16}$ realizaram um estudo envolvendo 1.507 crianças com idade entre 0 e 16 anos submetidas à anestesia geral com o objetivo de determinar a prevalência de tremores assim como as causas desencadeantes e as implicações clínicas. Após o procedimento cirúrgico a criança era admitida na sala de recuperação pós-anestésica e recebia oxigênio por máscara facial. Mediu-se a temperatura timpânica e registraram-se o tipo e a duração da intervenção cirúrgica, o método de indução anestésica (inalatório através de sevoflurano ou venosa com tiopental), a idade e a associação à peridural caudal. A temperatura da sala cirúrgica e da SRPA foi mantida entre $22^{\circ} \mathrm{C}$ e $23^{\circ} \mathrm{C}$. Dessas 1.507 crianças, apenas $53(3,5 \%)$ apresentaram tremores. Crianças com e sem tremores diferiram significativamente quanto ao tempo cirúrgico, temperatura corporal e idade. $\mathrm{O}$ tipo de operação e o sexo não apresentaram relação com a prevalência de tremores. $O$ risco de tremores aumentou com a idade acima de 6 anos, uso de agentes venosos na indução e tempo cirúrgico superior a 40 minutos. Trinta e sete de 53 pacientes apresentaram melhora dos tremores apenas com o aquecimento; 16 crianças necessitaram de meperidina $\left(0,35 \mathrm{mg} \cdot \mathrm{kg}^{-1}\right)$ por via venosa. Nesse estudo, a prevalência de tremor nas crianças que receberam tiopental na indução foi comparativamente maior. Entretanto, a idade mais avançada dessas crianças pode ter sido um fator de risco contribuinte. A freqüência de tremores foi, sob o aspecto estatístico, mais baixa em crianças que receberam peridural caudal.

\section{PREVENÇÃO E TRATAMENTO EM CRIANÇAS}

Kranke e col. ${ }^{3}$ propuseram um algoritmo para prevenção farmacológica e tratamento de tremores em crianças com base em estudos em adultos e recomendaram evitar hipotermia. Quando ocorreu tremor, trataram com aquecimento e em casos refratários, sugeriram a administração de clonidina $\left(1,5 \mu \mathrm{g} \cdot \mathrm{kg}^{-1}\right)$ ou meperidina $\left(0,35 \mu \mathrm{g} \cdot \mathrm{kg}^{-1}\right)$, ou seja, como o tratamento dos tremores é simples, eficaz e de baixo custo, e como a profilaxia é recomendada apenas em pacientes de alto risco, esta não deve ser feita rotineiramente em crianças. Em seu estudo, Akin e col. ${ }^{16}$ consideraram desnecessária a profilaxia de tremor em crianças, haja vista sua baixa incidência $(3,5 \%)$; devendo ser tratado apenas quando ocorrer.

\section{CONCLUSÃO}

A profilaxia de tremores pós-operatórios é simples e efetiva, a questão é avaliar quando devem ser prevenidos e quando devem ser tratados. $O$ tratamento dos tremores também é efetivo, porém muitos pacientes apresentaram recorrência do problema. $O$ fato de que manter a temperatura corporal normal tem um grande efeito nos tremores pós-operatórios põe em questão o uso profilático de fármacos. Parece que o seu uso preventivo deve ser feito apenas em casos especiais, como nos pacientes em que a maior demanda de consumo de oxigênio os coloque em risco de complicações ${ }^{65}$. Em estudo comparando vários fármacos isolados para a prevenção de tremores, a clonidina, a meperidina, o tramadol e o nefopam foram mais efetivos que o placebo e, embora a clonidina pudesse apresentar maior incidência de bradicardia, hipotensão arterial e sedação, esses efeitos não foram observados nas doses empregadas.

A incidência de tremores pode ser prevista com poder moderado de discriminação utilizando quatro fatores de risco derivados de uma análise de regressão logística: idade (a variável com maior poder preditivo), temperatura central na admissão na sala de recuperação pós-anestésica, intervenção cirúrgica prolongada e procedimento ortopédico. 
A profilaxia farmacológica de tremores pós-operatórios é possível e só será efetiva quando o risco for muito alto. Isso leva à questão se o tremor deve ser prevenido ou tratado. $\mathrm{O}$ tratamento com meperidina ou clonidina é muito eficaz. A opção por profilaxia administraria fármacos sem necessidade a muitos pacientes, expondo-os aos efeitos adversos. $O$ fato do aquecimento corporal intra-operatório e da manutenção da temperatura central ter grande efeito na incidência de tremores pós-operatórios ${ }^{10}$ coloca em questão a utilidade da profilaxia farmacológica. Casos especiais são os pacientes com suprimento cardíaco de oxigênio comprometido; neles pode ser preferível a profilaxia farmacológica e a clonidina deveria ser a escolha racional, pois, além de seu efeito antitremor, há um efeito favorável sobre o coração ${ }^{79}$.

\section{Intra- and Postoperative Tremors: Pre- vention and Pharmacological Treatment}

Viviane Ferreira Albergaria, TSA, M.D.; Michelle Nacur Lorentz, TSA, M.D.; Frederico Augusto Soares de Lima, M.D.

\section{INTRODUCTION}

In homeothermic species, the thermal regulation system coordinates the defenses against cold and heat, while maintaining internal body temperature within a narrow range, ideal for physiological and metabolic functions. However, the combination of anesthesia and exposure to the cold, can lead to hypothermia and tremors in surgical patients ${ }^{1,2}$. Since those tremors are usually self-limiting, do not become chronic, and normally do not have more serious consequences, they are often underestimated.

Postoperative tremors are frequently associated with loss of body heat, although hypothermia by itself does not fully explain them. However, they cause discomfort for the patients and sometimes can lead to complications ${ }^{3}$.

Along with nausea and vomiting, tremors are one of the most frequent problems during the initial recovery after general anesthesia 4,5 . Tremors are very uncomfortable and psychologically stressing. They can also lead to complications, especially in patients with coronary artery disease, due to an increase in oxygen consumption $\left(\mathrm{O}_{2}\right)$ by $100 \%$ to $600 \%$, increase production of carbon dioxide $\left(\mathrm{CO}_{2}\right)$, resulting in reduced minute ventilation, increased cardiac output ${ }^{6}$, increase in circulating catecholamines (increased heart rate), besides decreasing $\mathrm{O}_{2}$ saturation in mixed venous blood ${ }^{7-9}$. There can also be an increase in intracranial and intraocular pressures, interference with the ECG, pulse oximetry ${ }^{10}$, blood pressure, increase in metabolism, and cause lactic acidosis ${ }^{7-9}$.

Postoperative tremors can be of two types: the first corresponds to the tremor associated with thermal regulation that is associated with vasoconstriction, being a physiological response to central or peripheral hypothermia ${ }^{11,12}$ that develops in the intraoperative period; the second corresponds to the tremor associated with vasodilation or tremor not associated with thermal regulation ${ }^{13}$. It has been observed that tremors do not affect only hypothermic patients ${ }^{14,15}$ and they can also be present in patients whose temperature is normal 2,16.17 and, on the other hand, many hypothermic patients do not develop tremors. Sessler et al. concluded that postoperative tremors are secondary to thermal regulation ${ }^{12}$, are caused by hypothermia, and can also affect normothermic patients who are developing fever ${ }^{15}$. However, some tremors during labor ${ }^{18}$ and general anesthesia ${ }^{19}$ are not related with thermal regulation, and worsen due to undertreated pain 8,20 . More than ten years ago, Crossley ${ }^{21}$ enumerated some variables that influence the development of tremors: prolonged surgical procedures, male gender, anticholinergic agents (atropine), spontaneous ventilation, altered physical state (high ASA), more frequent in general surgical procedure than orthopedics, and blood transfusion. He also mentioned that advanced age, and the administration of propofol, alfentanil, or morphine were the most important variables in the protection against tremors.

\section{PHYSIOPATHOLOGY}

Several studies suggest that surgery-related factors, as pain and stress, contribute to the genesis of tremors ${ }^{12}$. The mechanisms involved with the development of tremors are complex and presumably result from adaptive evolution. There is not one pathway responsible for them; on the contrary, several mechanisms are capable to modulate thermal regulation responses ${ }^{15}$.

Intraoperative hypothermia, besides causing severe complications, may prolong hospitalization ${ }^{22}$, and can also be associated with a reduction in drug metabolism, delayed awakening from anesthesia, negative nitrogen balance, and tremors ${ }^{23}$.

The use of hypothermia as a therapeutic strategy for neuroprotection is still controversial ${ }^{24}$. Hypothermia reduces intracranial pressure, increases cerebral perfusion pressure and decreases its metabolism. However, even moderate hypothermia has undesirable side effects, such as tremors and peripheral vasoconstriction, resulting in hemodynamic instability, increase in oxygen consumption, and cerebral and myocardial ischemia.

In humans, moderate hypothermia results in better neurological recovery in patients who survive cardiorespiratory arrest ${ }^{25,26}$. The International Liaison Committee on Resuscitation (ILCOR) recommends its use in those cases ${ }^{27}$.

Postoperative tremors affect $5 \%$ to $65 \%$ of patients recovering from general anesthesia ${ }^{7}$, and $30 \%$ of those undergoing epidural anesthesia ${ }^{28}$. They can be tremors associated with thermal regulation or due to the release of cytokines caused by the surgical procedure. Core temperature usually falls 
from $0.5^{\circ} \mathrm{C}$ to $1.5^{\circ} \mathrm{C}$ in the first hour after anesthetic induction. The hypothermia that develops in the first hour after induction of general ${ }^{29}$ or neuroaxial ${ }^{30}$ anesthesia results primarily from the redistribution of heat from the center to the periphery. Every anesthetic, opioid, and sedative, reduces vasoconstriction and the autonomic control of thermal regulation (tremor threshold), facilitating the development of hypothermia.

In a study of 2,595 adult patients, Crossley ${ }^{21}$ detected tremors in 164 (6.5\%) and identified male gender and preanesthetic medication with anticholinergics as risk factors. The type of anesthetic induction was not considered a risk factor in this study. Another study by Eberhat et al. ${ }^{31}$ evaluated 1,340 consecutive patients who underwent surgery under general anesthesia, and observed tremors in $11.9 \%$ of them. Risk factors related with postoperative tremors were hypothermia, age, orthopedic surgeries, and prolonged surgical procedures; but age seemed the most important factor. The objective of that study was to develop an algorithm to anticipate tremors; patients undergoing surgical procedures were evaluated, excluding those who presented preoperative hypothermia or those who used drugs that could interfere with thermal regulation (clonidine, meperidine, phenothiazine). The incidence of moderate or severe tremor (grades 3 and 4 ) was $11.6 \%$. The total incidence of any tremor (including grade 2) was $14.4 \%$. Thermal regulation activity (including vasoconstriction and piloerection) was observed in $17.6 \%$. Among these patients, only $33 \%$ complained of being cold. The work used multivariable analysis and found three independent factors that predict the development of tremors: youth, surgeries with endoprosthesis, and low core body temperature. Age was by far the most important risk factor, being responsible for more than $70 \%$ of the predictive power of the model. This was not surprising, because thermal regulation response is decreased in the elderly ${ }^{15}$. It is difficult to determine independent risk factors for tremors before surgery, since several variables influence postoperative thermal regulation in the case of volatile ${ }^{32}$ or intravenous anesthetics, and sedatives ${ }^{33}$. Besides, almost every drug used regularly by patients (antihypertensives, those used in the treatment of diabetes, and coumarin derivatives) seems to offer significant protection against tremors. In fact, the incidence of tremors is decreased in patients taking propranolol ${ }^{34}$.

Core temperature contributes $30 \%$ to the control of tremors and vasoconstriction, while mean skin temperature contributes $20 \%{ }^{35}$. Therefore, it is reasonable to assume that, in the absence of a surgical procedure, hypothermia contributes for the development of tremors and that normothermia protects against it ${ }^{12}$. The study demonstrated that core temperature had only a slight influence on the development of tremors when compared with age (most important factor), and was compatible with the results of other studies that demonstrated that temperature has a weak predictive power for the development of tremors ${ }^{36,37}$. According to the analysis of multifactorial logistic regression, core temperature was not directly related with postoperative tremors in children, while the relative change of perioperative temperature was one of three independent predictors ${ }^{38}$. However, tremors in patients whose body temperature is lower than $36^{\circ} \mathrm{C}$ lasted longer than in those who were warmed up ${ }^{39}$.

The study confirmed a previous work ${ }^{21}$ that showed that orthopedic surgeries (especially endoprosthesis) using bone cement was an independent risk factor for the development of tremors. A possible explanation would be the release of cytokines (tumor necrosis factor-alpha and interleukin-6) stimulated by the bone cement polymethylmethacrylate ${ }^{40}$. Prolonged surgeries were associated with a higher incidence of tremors. A possible explanation is that these procedures are more complex and invasive. Damaged tissue might release pyrogenic substances that change the trigger point of the thermal regulation system in the postoperative period, leading to the development of tremors ${ }^{41}$.

Active intraoperative warming to maintain normothermia is important in the prevention of tremors ${ }^{42,43}$. The center of thermal regulation is in the posterior hypothalamus, and its activity is modulated by temperature receptors in the skin, visceras, and in several levels of the neuroaxis ${ }^{42,44}$. Every volatile anesthetic produces tremors by changing the tremor threshold 45,46 .

During anesthesia, the patient has no thermal regulation responses, because the reflex is inhibited. During recovery, the inhibition disappears and tremors appear when the temperature is below the thermal regulation threshold ${ }^{47}$.

\section{TREMORS AND ANESTHESIA}

Since hypothermia is one of the predictive factors for the development of tremors, and being an independent risk factor for cardiac morbidity ${ }^{48}$, it should be avoided, recognized, and treated. Although it might be beneficial during the intraoperative period, by decreasing metabolism and protecting vital organs from ischemia, when the patient awakes from anesthesia, adrenergic and metabolic responses of thermal regulation are activated, causing postoperative discomfort, tremors, increased bleeding, and increased incidence of infection and cardiovascular effects ${ }^{49}$. During the intraoperative period, the incidence of myocardial ischemia and ventricular tachycardia are similar in hypothermic and normothermic patients. On the other hand, during the postoperative period, the incidence of ventricular tachycardia and electrocardiographic changes is greater in hypothermic patients ${ }^{48}$. The disparity between the intra- and postoperative periods suggests that anesthesia might have a protective effect against the adverse responses to the stress caused by the cold. There are two response mechanisms of thermal regulation to hypothermia that might be harmful to the cardiovascular system: the adrenergic response with vasoconstriction associated with elevation in blood pressure, and increased metabolic demand related to tremors ${ }^{50,51}$. Hemodynamic 
responses to the vasoconstriction secondary to thermal regulation seem to be similar without anesthesia and with different concentrations of desflurane and isoflurane ${ }^{52}$.

\section{CLASSIFICATTION OF TREMORS}

The degree of tremors was evaluated and classified according to a qualitative scale in the work of Crossley and Mahajan ${ }^{36}$ : 0 = without tremor; $1=$ without visible muscular activity but with piloerection, peripheral vasoconstriction, or both; 2 = muscular activity affecting only one muscle group; 3 = moderate muscular activity involving more than one muscle group, but without generalized tremors; and $4=$ severe muscular activity involving the entire body.

\section{PHARMACOLOGICAL PREVENTION OF TREMORS}

Proper warming of the body is not always feasible. Therefore, tremors should be prevented pharmacologically in selected patients.

Several drugs have been studied for the prophylaxis and treatment of tremors, including clonidine, meperidine, ketanserin (an anti-hypertensive drug, and antagonist with high affinity for 5-HT and $\alpha_{1}$-adrenergic receptors); doxapram (respiratory stimulant); and tramadol and other opioids ${ }^{15,53}$. The cholinergic system is probably among the mediators of tremors. Physostigmine, an anticholinesterase drug that crosses the blood-brain barrier, is widely used to treat the central cholinergic syndrome ${ }^{54}$. Studies suggest that physostigmine increases the cholinergic neurotransmission by stimulating the hypothalamus-hypophysis-adrenal axis and the medulla of the adrenal gland, inhibiting generalized thermal regulation.

Moderate hypothermia may have undesirable effects, such as tremors and vasoconstriction, and measures to prevent them in the postoperative period might be necessary. There are reports demonstrating that clonidine reduces the incidence of postoperative tremors, but the mechanism of action has not been elucidated ${ }^{54}$. The $\alpha_{2}$-agonists exert their action on $\alpha_{2}$ - adrenergic and imidazolic receptors. The first ones can be found in the brain, spinal cord, and the periphery. In the brain, they are related with sedation; in the spinal cord, with analgesia. The effects of clonidine on postsynaptic $\alpha_{2}$-receptors in the central nervous system reduces the sympathetic tonus that leads to peripheral vasodilation and hypotension ${ }^{24}$. The effects of meperidine on thermal regulation seem to involve $\alpha_{2}$-adrenergic receptors ${ }^{41}$.

Several drugs (meperidine, ketanserin, sufentanil, alfentanil, tramadol, physostigmine, urapidil, nefopam, doxapram, and nalbuphine) have already been tested to prevent or treat tremors ${ }^{7-9,28,55,56}$. Meperidine proved to be one of the most effective ${ }^{56,57}$, probably by acting on the thermal regulation center ${ }^{55}$ or through opioid receptors ${ }^{15}$. It is possible that NMDA receptors also modulate thermal regulation in several levels ${ }^{15}$. Ketamine, a NMDA antagonist, showed to be capable to inhibit tremors ${ }^{58}$.

Treatments established to prevent tremors include several drugs, such as nefopam, a benzoxazocin, that inhibits the reuptake of noradrenaline, dopamine, and serotonin. These drugs are not specific for tremors and exert their action through different mechanisms, such as decreasing the activation of the sympathetic nervous system ${ }^{9,53,59}$, adrenal suppression ${ }^{38}$, stimulation of opioid receptors, or influencing the serotoninergic system $5,9,15,60$.

The cholinergic system is an important pathway that can potentially influence tremors. The hypothalamus and the striated body are the regions in the brain in which physostigmine is more active ${ }^{61,62}$. Physostigmine is a central acting cholinesterase inhibitor that interacts with cholinergic muscarinic, serotoninergic, and endorphinergic brain receptors, as well as with $\mu$-opioid agonists and $\alpha_{2}$-agonists ${ }^{15}$. Therefore, it seems to work by increasing central neurotransmission. It seems to be safe and highly effective, with very little side effects that include hemodynamic changes; however, they were not observed in the study, probably due to the slow administration (15 minutes) ${ }^{62,63}$.

Besides, physostigmine produced analgesia by releasing $\beta$-endorphins ${ }^{64}$; and postoperative pain can lead to tremors ${ }^{20}$. The dose of physostigmine is usually $2 \mathrm{mg}{ }^{62,63}$. Physostigmine reduced significantly the incidence and severity of tremors, as much as nefopam. Cholinergic pathways seem to be involved in the thermal regulation, decreasing tremors without influencing awakening from anesthesia, alertness, and postoperative recovery.

In another study, divided in two stages, Stapelfeldt et al. ${ }^{24}$ investigated the use of clonidine in 48 patients undergoing elective neurosurgery. First, they determined, in 14 patients, the dose of clonidine necessary to prevent postoperative tremors after inducing moderate hypothermia $\left(35^{\circ} \mathrm{C}\right)$. This dose was $3 \mu \mathrm{g} \cdot \mathrm{kg}^{-1}$. In the second stage, they investigated whether clonidine, due to its sedative action, would delay awakening from anesthesia in neurosurgeries and, therefore, delay the neurological evaluation. Thirty-four patients who underwent neurological surgeries were randomly distributed in two groups to receive clonidine ( $\left.3 \mu \mathrm{g} . \mathrm{kg}^{-1}\right)$ or normal saline when the dura mater began to be sutured. Inhalational anesthetics and the infusion of opioids were discontinued at that moment and propofol was administered to every patient. Recovery of patients was evaluated two hours after the end of anesthesia at the following moments: upon opening his/ her eyes, when extubated, regarding obeying commands and orientation in time and space. There were no significant differences between both groups in the duration of postanesthetic recovery. Systolic blood pressure was lower in the clonidine group, which was statistically significant; however, it was not clinically significant ${ }^{24}$. The ideal dose of clonidine has not been determined yet, but small doses may be enough to prevent tremors after moderate hypothermia. 
Piper et al. ${ }^{9}$ compared clonidine and nefopam in the prevention of tremors and found out that they are equally effective. However, clonidine caused more sedation and delayed awakening, suggesting that nefopam is a better prophylactic drug.

In another systematic revision, Kranke et al. ${ }^{65}$ described the efficacy and risks of using drugs to prevent the development of tremors. They analyzed 27 articles (1,348 adults received medication and 931 were controls). The incidence of tremors in the control group was extremely frequent (52\%). Clonidine, 65 to $300 \mu \mathrm{g}$ (1,078 patients), 12.5 to $35 \mathrm{mg}$ of meperidine (250 patients), 35 to $220 \mathrm{mg}$ of tramadol (250 patients), and 6.5 to $11 \mathrm{mg}$ of nefopam (204 patients) were tested in at least three studies each. All of them were more effective when compared with the controls. The studies suggest that less than four patients have to receive prophylactic clonidine in order that one patient does not present tremors after the surgery. For meperidine, nefopam, and tramadol, the efficacy seemed even more convincing. The high prevalence of tremors in patients who were not treated suggests that study populations have a high risk, and might not be representative of the general population. Besides, the high prevalence in this population tends to overestimate the usefulness of therapeutic interventions. Clonidine was tested in more studies than the other drugs, which allowed the evaluation of its dose and the time of administration. To be more effective, it should be administered during induction (and not before) in doses greater than $140 \mu \mathrm{g}{ }^{65}$.

There were limiting factors. First, the mechanism of action against tremors of the drugs analyzed is not completely characterized. Some of them might change the tremor threshold. This has been demonstrated for meperidine ${ }^{66}$ and clonidine ${ }^{67}$. Second, it was not possible to establish predictive factors for postoperative tremors. Third, like a similar previous study ${ }^{41}$, it was necessary to use indirect comparisons to estimate the relative efficacy of the anti-tremor interventions, since there is no gold standard that can be used as a comparison. Fourth, most of the studies had a small cohort, and only 5 of them had 50 patient or more ${ }^{68,71}$, and studies with small samples tend to overestimate the efficacy of the treatment. Fifth, the quality of the method used in most of the studies was not satisfactory. And sixth, the report on side effects was poor, but it does not necessarily mean that they were not present. Dal et al. ${ }^{72}$ compared low doses of ketamine, meperidine, and placebo in the prophylaxis of tremors. Ninety patients, ages 18 to 65 years, physical status ASA I or II, who underwent general anesthesia for 60 to 180 minutes, were divided in 3 groups of 30 each. Group $S$ received normal sa-

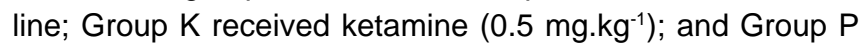
received meperidine $(20 \mathrm{mg}) 20$ minutes before surgery. The tympanic temperature of every patient was greater than $36^{\circ} \mathrm{C}$ and, therefore, did not require warming. The incidence of tremors was significantly lower in Groups $\mathrm{K}$ and $\mathrm{P}$ than in Group S, and there were no differences between Groups K and $P$. The length of time until the first request of analgesics in Group S was much smaller (12 $\mathrm{min}$ ) than in Groups K (25 $\mathrm{min}$ ) and $\mathrm{P}(32 \mathrm{~min})$. Two hours after the end of the procedure, every patient needed analgesics. There were no differences in pain scores among the groups, using the Visual Analogic Scale (VAS), 30 minutes after the end of the surgery. Two patients in Group K had grade 2 tremors and were treated with meperidine, which was effective, probably because its mechanism of action is different, or because the dose of $0.5 \mathrm{mg} . \mathrm{kg}^{-1}$ of ketamine was not enough. Patients did not present adverse effects related to ketamine. This drug might be an alternative for the prophylaxis of postoperative tremors in patients who cannot receive meperidine. Future studies might demonstrate the ideal dose of ketamine for the prophylaxis of postoperative tremors. There were no differences between Groups $\mathrm{K}$ and $\mathrm{P}$ regarding the prevention of tremors. There was no relationship between axillary temperature and the development of tremors ${ }^{58}$.

Ketamin is a NMDA antagonist that is effective in the prevention of tremors, probably through the thermogenesis without tremors pathway, by acting on the hypothalamus, or by a $\beta$-adrenergic effect, besides having some effect on the $\kappa$ receptor. A study suggested that the action of meperidine against tremors might be through $\kappa$ receptors, because high doses of naloxone inhibits its action (high doses inhibit mu and kappa receptors), while small doses of naloxone do not (inhibit only mu receptors) ${ }^{56}$. The disadvantage of meperidine is the risk of respiratory depression, nausea, and vomiting.

Rohm et al. ${ }^{73}$ investigated the effects of physostigmine in the prophylaxis of postoperative tremors, and compared it with nefopam, a drug known to be effective ${ }^{5}$. Eighty-nine patients undergoing abdominal or urological surgeries were evaluated. Patients received $2 \mathrm{mg}$ of physostigmine $(n=31), 10 \mathrm{mg}$ of nefopam $(n=30)$, or normal saline $(n=28)$. Hemodynamic parameters and temperature were measured at induction and $5,15,30$, and 60 minutes after arrival in the recovery room. The incidence of tremors was significantly lower after the administration of physostigmine and nefopam $(9.7 \%$ and $3.3 \%$ ) than with placebo (53.6\%). Time of extubation, hemodynamic parameters, and tympanic temperature were similar in all groups. Only nefopam reduced significantly the incidence of postoperative nausea and vomiting. Physostigmine reduced successfully and safely the incidence and severity of tremors. This success was similar to that of nefopam and it did not delay the recovery of patients.

Another study was undertaken by Hong and Lee ${ }^{74}$ with patients undergoing cesarean sections with epidural anesthesia with $8 \mathrm{mg}$ to $10 \mathrm{mg}$ of $0.5 \%$ hyperbaric bupivacaine associated with morphine $(0.1 \mathrm{mg}$ or $0.2 \mathrm{mg})$, meperidine (10 mg), or just local anesthetic (LA). The incidence of tremors was greater in patients who received just LA (23.3.\%). Those who received meperidine had not only the lowest incidence (3.3\%), but tremors were also less severe when compared with those who received morphine $(0.1 \mathrm{mg}-17 \% ; 0.2 \mathrm{mg}-$ $13.3 \%)$. The epidural administration of opioids can also be capable of abolishing tremors, although their mechanism of 
action, influencing tremor threshold, has not been established ${ }^{75}$. A study by Abreu et al. ${ }^{75}$ compared the incidence of intra- and postoperative tremors in epidural blocks with and without fentanyl. They concluded that adding $100 \mu \mathrm{g}$ of fentanyl to the LA for the epidural block does not abolish the tremor, but reduces the incidence and severity, without increasing the incidence of nausea and vomiting.

\section{NON-PHARMACOLOGICAL PREVENTION OF TREMORS}

Tremors can be treated by warming up the patient, applying radiant heat, or drugs ${ }^{55}$.

Among the measures used in the maintenance of body temperature, the warm air blanket can be beneficial and cost effective ${ }^{43}$.

Simply covering the patient with a blanket was indicated as a tremor-reducing factor that did not change core temperature ${ }^{43}$. Skin temperature is not a predictive factor for tremors. However, it is unquestionable that increasing skin temperature enough can, alone, stop tremors.

\section{TREATMENT OF POSTOPERATIVE TREMORS}

The development of tremors is a frequent complication in the recovery room, and several drugs are used to treat it, although their mechanism of action have not been elucidated ${ }^{41}$; in a meta-analysis by Kranke et al., meperidine was deemed the most effective drug for the treatment of tremors, and a dose of $25 \mathrm{mg}$ reduced the recurrence of tremors nine times as often as placebo, maintaining an anti-tremor effect for at least 45 minutes. Meperidine was associated with a small increase in side effects, such as nausea, vomiting, and reduction in respiratory rate. In situations that meperidine cannot be used, the authors suggest the use of clonidine $(150 \mu \mathrm{g})$, ketanserin $(10 \mathrm{mg})$, alfentanil $(0.25 \mathrm{mg})$, or doxapram $(100 \mathrm{mg})$. It should be mentioned that the intraoperative maintenance of body temperature and the use of warm air blankets decreased the need of drugs for the treatment of tremors ${ }^{76}$.

In a study with 220 patients aiming at identifying the effective dose of meperidine for the treatment of tremors, Crossley ${ }^{76}$ concluded that $0.35 \mathrm{mg} \cdot \mathrm{kg}^{-1}$ is the smallest dose necessary to treat adequately postoperative tremors.

Tramadol has also been used in the past few years to treat tremors and, according to some studies, its efficacy is similar to that of meperidine ${ }^{77}$.

Nefopam is a central-acting analgesic benzoxacin that reduces tremors without affecting ventilation, with minimal sedation, and proven efficacy. Contrary to other drugs, it does not cause vasoconstriction, working only in the tremor pathway ${ }^{78}$.

\section{TREMORS IN PEDIATRIC PATIENTS}

Most of the studies on postoperative tremors are done in adults and, when they are done with children, they include a small number of patients ${ }^{16}$. A classical work by Akin et al. ${ }^{16}$ with 1,507 children ages 0 to 16 years, undergoing general anesthesia, was undertaken to determine the prevalence of tremors, as well as the triggers and clinical implications. After the surgery, the child was transferred to the recovery room and received oxygen by face mask. The tympanic temperature was measured, the type and duration of the surgery were recorded, as well as the method of anesthetic induction (inhalational, with sevoflurane, or intravenous, with thiopental), age, and association with caudal epidural. The temperature of the operating room and recovery room was maintained between $22^{\circ} \mathrm{C}$ and $23^{\circ} \mathrm{C}$. Of 1,507 children, only $53(3.5 \%)$ developed tremors. There was a statistically significant difference between children with and without tremors regarding length of surgery, body temperature, and age. The type of surgery and gender had no relationship with the prevalence of tremors. The risk of tremors increased with ages above 6 years, use of intravenous agents for induction, and surgeries lasting more than 40 minutes. In 37 out of the 53 patients, tremors improved by rewarming alone; 16 of them needed intravenous meperidine $\left(0.35 \mathrm{mg} \cdot \mathrm{kg}^{-1}\right)$. In this study, the prevalence of tremors in children who received thiopental for induction was significantly greater. However, since these children were older, that could have been a contributing factor. The incidence of tremors was statistically lower in children who received caudal epidural anesthesia.

\section{PREVENTION AND TREATMENT OF TREMORS IN CHILDREN}

Kranke et al. ${ }^{3}$ proposed an algorithm for the pharmacological prevention and treatment of tremors in children based on studies done in adults, and recommended that hypothermia should be avoided. When a child developed tremor, he/she was treated by rewarming and, if there was no response, they suggested the administration of clonidine $\left(1.5 \mu \mathrm{g} . \mathrm{kg}^{-1}\right)$ or meperidine $\left(0.35 \mu \mathrm{g} . \mathrm{kg}^{-1}\right)$ that is, since the treatment of tremors is simple, effective, and low cost, and since prophylaxis is recommended only in high risk patients, it should not be routinely done in children. In their study, Akin et al. ${ }^{16}$ did not consider that prophylaxis of tremors in children was necessary, due to the low incidence (3.5\%), and it should be treated when present.

\section{CONCLUSION}

Prophylaxis of postoperative tremors is simple and effective. The problem is to evaluate when they should be prevented or treated. Treatment of tremors is also effective; however, many patients presented recurrence of the problem. Since the maintenance of a normal body temperature influences profoundly postoperative tremors, the use of prophylactic drugs is questioned. It seems they should be done only in special cases, such as patients in whom greater oxygen consumption would increase the risk of complications ${ }^{65}$. 
In a study comparing several drugs isolatedly to prevent tremors, clonidine, meperidine, tramadol, and nefopam were more effective than placebo and, although the incidence of bradycardia, hypotension, and sedation are greater with clonidine, these effects were not observed in the doses used in this study.

The incidence of tremors can be predicted with moderate discriminative power using four risk factors derived from a logistic regression analysis: age (variable with the greatest predictive power), core temperature upon admission to the recovery room, prolonged surgical procedure, and orthopedic procedures.

The pharmacological prophylaxis of postoperative tremors is possible and will only be effective when the risk is too high. This raises the question whether tremors should be prevented or treated. Treatment with meperidine or clonidine is very effective. The option for prophylaxis means the unnecessary administration of drugs to several patients, exposing them to side effects. Since intraoperative body warming and maintenance of core temperature greatly influence the incidence of postoperative tremors ${ }^{10}$, the use of pharmacological prophylaxis is questioned. Patients in whom the cardiac supply of oxygen is compromised are a special case; in these cases, pharmacological prophylaxis is preferable, and clonidine should be the rational choice because, besides the anti-tremor effect, it has a favorable effect on the heart ${ }^{79}$.

\section{REFERÊNCIAS - REFERENCES}

01. Frank SM, Beattie C, Christopherson R et al. - Epidural versus general anesthesia, ambient operation room temperature, and patient age as predictors of inadvertent hypothermia. Anesthesiology, 1992;77:252-257.

02. Kurz A, Sessler DI, Narzt E et al. - Postoperative hemodynamic and thermoregulatory consequences of intraoperative core hypothermia. J Clin Anesth, 1995;7:359-366.

03. Kranke P, Eberhart LH, Roewer $\mathrm{N}$ et al. - Postoperative shivering in children: a review on pharmacologic prevention and treatment. Paediatr Drugs, 2003;5:373-383.

04. Buggy D, Higgins $P$, Moran $C$ et al. - Clonidine at induction reduces shivering after general anaesthesia. Can $\mathrm{J}$ Anaesth, 1997;44:263-267.

05. Piper SN, Röhm KD, Suttner SW et al. - A comparison of nefopam and clonidine for the prevention of postanaesthetic shivering: a comparative, double-blind and placebo-controlled dose-ranging study. Anaesthesia, 2004;59:559-564.

06. Ciofolo MJ, Clergue F, Devilliers $C$ et al. - Changes in ventilation, oxygen uptake, and carbon dioxide output during recovery from isoflurane anesthesia. Anesthesiology, 1989;70:737-741.

07. Singh P, Dimitriou V, Mahajan RP et al. - Double-blind comparison between doxapram and pethidine in the treatment of postanaesthetic shivering. Br J Anaesth, 1993;71:685-688.

08. Mathews S, Al Mulla A, Varghese PK et al. - Postanaesthetic shivering - a new look at tramadol. Anaesthesia, 2002;57:394398.

09. Piper SN, Suttner SW, Schimdt CC et al. - Nefopam and clonidine in the prevention of postanaesthetic shivering. Anaesthesia, 1999;54:695-699.
10. Buggy DJ, Crossley AW - Thermoregulation, mild perioperative hypothermia and postanaesthetic shivering. Br J Anaesth, 2000; 84:615-628.

11. Lienhart A, Fiez N, Deriaz $\mathrm{H}$ - Frisson postoperatoire: analyse des principaux facteurs associes. Ann Fr Anesth Reanim, 1992; 11:488-495.

12. Sessler DI, Rubinstein EH, Moayeri A - Physiologic responses to mild perianesthetic hypothermia in humans. Anesthesiology, 1991;75:594-610.

13. Horn EP - Postoperative shivering; aetiology and treatment. Curr Opin Anaesthesiol, 1999;12:449-453.

14. Camus $Y$, Delva $E$, Just $B$ et al. - Leg warming minimizes core hypothermia during abdominal surgery. Anesth Analg, 1993; 77:995-999.

15. De Witte J, Sessler DI - Perioperative shivering: physiology and pharmacology. Anesthesiology, 2002;96:467-484.

16. Akin A, Esmaoglu A, Boyaci A - Postoperative shivering in children and causative factors. Paediatr Anaesth, 2005; 15:1089-1093.

17. Crossley AW - Peri-operative shivering. Anaesthesia, 1992; 47:193-195.

18. Panzer O, Ghazanfari N, Sessler DI et al. - Shivering and shivering-like tremor during labor with and without epidural analgesia. Anesthesiology, 1999;90:1609-1616.

19. Horn EP, Sessler DI, Standl T et al. - Non-thermoregulatory shivering in patients recovering from isoflurane or desflurane anesthesia. Anesthesiology, 1998;89:878-886.

20. Horn EP, Schroeder F, Wilhelm S et al. - Postoperative pain facilitates nonthermoregulatory tremor. Anesthesiology, 1999; 91:979-984.

21. Crossley AW - Six months of shivering in a district general hospital. Anaesthesia, 1992;47:845-848.

22. Alfonsi A, Nourredine KE, Adam F et al. - Effect of postoperative skin-surface warming on oxygen consumption and the shivering threshold. Anaesthesia, 2003;58:1228-1234.

23. Sessler DI - Mild perioperative hypothermia. New England J Med,1997;336:1730-1737.

24. Stapelfeldt C, Lobo EP, Brown R et al. - Intraoperative clonidine administration to neurosurgical patients. Anesth Analg, 2005; 2005:226-232.

25. Bernard SA, Gray TW, Buist MD et al. - Treatment of comatose survivors of out-of-hospital cardiac arrest with induced hypotermia. N Engl J Med, 2002;346:557-563.

26. Hypothermia after Cardiac Arrest Study Group - Mild therapeutic hypothermia to improve the neurologic outcome after cardiac arrest. N Engl J Med, 2002;346:549-556.

27. Nolan JP, Morley PT Vanden Hock TL et al. - Therapeutic hypothermia after cardiac arrest: an advisor statement by the Advanced Life Support Task Force of the International Liaison Committee on Resuscitation. Circulation, 2003;108:118-121.

28. Horn EP, Werner C, Sessler DI et al. - Late intraoperative clonidine administration prevents postanesthetic shivering after total intravenous or volatile anesthesia. Anesth Analg 1997; 84:613-617.

29. Matsukawa T, Sessler DI, Sessler AM et al. - Heat flow and distribution during induction of general anesthesia. Anesthesiology, 1995;82:662-673.

30. Matsukawa T, Sessler DI, Christensen R et al. - Heat flow and distribution during epidural anesthesia. Anesthesiology, 1995; 83:961-967.

31. Eberhart LH, Doderlein F, Eisenhardt G et al. — Independent risk factors for postoperative shivering. Anest Analg, 2005;101:18491857.

32. Xiong J, Kurz A, Sessler DI et al. - Isoflurane produces marked and nonlinear decreases in the vasoconstriction and shivering thresholds. Anesthesiology, 1996;85:240-245. 
33. Matsukawa T, Kurz A, Sessler DI et al. - Propofol linearly reduces the vasoconstriction and shiverig thresholds. Anesthesiology, 1995;82:1169-1180.

34. Lee DS, Shaffer MJ - Low incidence of shivering with chronic propranolol therapy. Lancet, 1986;1:500.

35. Cheng C, Matsukawa T, Sessler DI et al. - Increasing mean skin temperature linearly reduces the core-temperature thresholds for vasoconstriction and shivering in humans. Anesthesiology, 1995;82:1160-1168.

36. Crossley AW, Mahajan RP - The intensity of postoperative shivering is unrelated to axillary temperature. Anaesthesia, 1994;49:205-207.

37. Holdcroft A, Hall GM - Heat loss during anaesthesia. $\mathrm{Br} \mathrm{J}$ Anaesth, 1978;50:157-164.

38. Lyons B, Taylor A, Power C et al. - Postanaesthetic shivering in children. Anaesthesia, 1996;51:442-445.

39. Vaughan MS, Vaughan RW, Cork RC - Postoperative hypothermia in adults: relationship of age, anesthesia, and shivering to rewarming. Anesth Analg, 1981;60:746-751.

40. Miyaguchi M, Kobayashi A, Iwaki $\mathrm{H}$ et al. - Human monocyte response to retrieved polymethylmethacrylate particles. J Biomed Mater Res, 2002;62:331-7.

41. Kranke P, Eberhart LH, Roewer $\mathrm{N}$ et al. - Pharmacological treatment of postoperative shivering: a quantitative systematic review of randomized controlled trials. Anesth Analg, 2002; 94:453-460.

42. Crossley AW - Postoperative shivering: the influence of body temperature. BMJ, 1995;311:764-765.

43. Buggy D, Hughes $\mathrm{N}-$ Pre-emptive use of the space blanket reduces shivering after general anaesthesia. Brit $\mathrm{J}$ Anaesth, 1994;72:393-396.

44. Gordon CJ, Heath JE - Integration and central processing in temperature regulation. Ann Rev Phsiol, 1986;48:595-612.

45. Sessler DI, Esrael D, Pozos RS et al. - Spontaneous postanesthetic tremor does not resemble thermoregulatory shivering. Anesthesiology, 1988;68:843-850.

46. Annadata R, Sessler DI, Tayefeh F et al. - Desflurane slightly increases the sweating threshold, but produces marked and non-linear decreases in the vasoconstriction and shivering thresholds. Anesthesiology, 1995;83:1205-1211.

47. Cheong KF, Low TC - Propofol and postanesthetic shivering. Anaesthesia, 1995;50:550-552.

48. Frank SM, Fleisher LA, Breslow MJ at al - Perioperative maintenance of normothermia reduces the incidence of morbid cardiac events: a randomized clinical trial. JAMA, 1997; 277:1127-1137.

49. Pestel GJ, Kurz A - Hypothermia: it's more than a toy. Curr Opin Anesthesiol, 2005;18:151-156.

50. Lopez M, Selsser DI, Walter K et al. - Rate and gender dependence of the sweating, vasoconstriction, and shivering thresholds in humans. Anesthesiology, 1994;80:780-788.

51. Macintyre PE, Pavlin EG, Dwersteg JF - Effect of meperidine on oxygen consumption, carbon dioxide production, and respiratory gas exchange in postanesthesia shivering. Anesth Analg, 1987;66:751-755.

52. Greif R, Laciny S, Rajek A et al. - Blood pressure response to thermoregulatory vasoconstriction during isoflurane and desflurane anesthesia. Acta Anaesthesiol Scand, 2003;47:847-852.

53. Piper SN, Maleck WH, Boldt J et al. - A comparison of urapidil, clonidine, meperidine and placebo in preventing postanesthetic shivering. Anesth Analg, 2000;90:954-957.

54. Milam SB, Bennett CR - Physostigmine reversal of druginduced paradoxical excitement. Int J Oral Maxillofac Surg, 1987; 16:190-193.

55. Vanderstappen I, Vandermeersch E, Vanacker B et al. - The effect of prophylactic clonidine on postoperative shivering: a large prospective double-blind study. Anaesthesia, 1996; 51:351-355

56. Wrench IJ, Cavill G, Ward JE et al. - Comparison between alfentanil, pethidine and placebo in the treatment of postanaesthetic shivering. Br J Anaesth, 1997;79:541-542.

57. Terasako K, Yamamoto $M$ - Comparison between pentazocine, pethidine and placebo in the treatment of post-anaesthetic shivering. Acta Anaesthesiol Scand, 2000;44:311-312.

58. Sharma DR, Thakur JR - Ketamine and shivering. Anaesthesia, 1990:45:252-253.

59. Delaunay L, Bonnet F, Liu N et al. - Clonidine comparably decreases the thermoregulatory thresholds for vasoconstriction and shivering in humans. Anesthesiology, 1993;79:470-474.

60. Powell RM, Buggy DJ - Ondansetron given before induction of anesthesia reduces shivering after general anesthesia. Anesth Analg, 2000;90:1423-1427.

61. Pappata S, Tavitian B, Traykov L et al. — In vivo imaging of human cerebral acetylcholinesterase. J Neurochem, 1996;67:876-879.

62. Horn EP, Standl T, Sessler DI et al. - Physostigmine prevents postanesthetic shivering as does meperidine or clonidine. Anesthesiology, 1998;88:108-113.

63. Latasch L, Müller B, Freye E - Routinemassige gabe von Physostigmin hat fur die postoperative phase keine bedeutungeine randomisierte untersuchung zur vigilans zum kreislaufverhalten, muskelzitten und schmerzmittelbedarf. Anasthesiol Intensivmed Notfallmed Schmerzth, 2003;38:528-537.

64. Risch SC, Janowsky DS, Mott MA et al. - Central and peripheral cholinesterase inhibition: effects on anterior pituitary and syjmpathomimetic function. Psychoneuroendocrinology, 1986; 11:221-230

65. Kranke $\mathrm{P}$, Eberhart LH, Roewer $\mathrm{N}$ et al. - Single-dose parenteral pharmacological interventions for the prevention of postoperative shivering: a quantitative systematic review of randomized controlled trials. Anesth Analg, 2004;99:718-727.

66. Kurz A, Ikeda T, Sessler DI et al. - Meperidine decreases the shivering threshold twice as much as the vasoconstriction threshold. Anesthesiology, 1997;86:1046-1054.

67. Nicolaou G, Chen AA, Johnston CE et al. - Clonidine decreases vasoconstriction and shivering thresholds, without affecting the sweating threshold. Can J Anaesth, 1997;44:636-642.

68. Eberhart LH, Novatchkov N, Schricker T et al. - Clonidin in vergleich zu midazolam zur intravenosen pramedikation von ambulatenten eingriffen. Eine kontrollierte doppelblindstudie bei ASA 1-patienten. Anasthesiol Intensivmed Notfallmed Schmerzther, 2000;35:388-393.

69. Sia S - I.V. clonidine prevents post-extradural shivering. $\mathrm{Br} \mathrm{J}$ Anaesth, 1998;81:145-146.

70. Vanderstappen I, Vandermeersch E, Vanacker B et al. - The effect of prophylactic clonidine on postoperative shivering: a large prospective double-blind study. Anaesthesia, 1996; 51:351-355.

71. Weinbroum AA, Geller E - Flumazenil improves cognitive and neuromotor emergence and attenuates shivering after halothane, enflurane and isoflurane-based anesthesia. Can J Anaesth, 2001;48:963-972.

72. Dal D, Kose A, Honca M et al. - Efficacy of prophylactic ketamine in preventing postoperative shivering. $\mathrm{Br} \mathrm{J}$ Anaesth, 2005; 95:189-192.

73. Rohm KD, Riechmann J, Boldt $\mathrm{J}$ et al. - Physostigmine for the prevention of postanaesthetic shivering following general anaesthesia - a placebo-controlled comparasion with nefopan. Anaesth, 2005;60:433-438.

74. Hong JY, Lee IH - Comparison of the effects of intrathecal morphine and pethidine on shivering after caesarean delivery under combined spinal epidural. Anaesthesia, 2005;60:1168-1172. 
75. Abreu MP, Vieira JL, Lutti MN, et al. - Incidência de tremor em anestesia com ou sem fentanil: estudo comparativo. Rev Bras Anestesiol, 2004;54:153-161.

76. Wrench IJ, Singh P, Dennis AR et al. - The minimum effective doses of pethidine and doxapram in the treatment of post-anaesthestic shivering. Anaesthesia, 1997;52:32-36.

77. Tsai YC, Chu KS - A comparation of tramadol, amitriptyline, and meperidine for postepidural anesthetic shivering in parturients. Anesth Analg, 2001;93:1288-1292.

78. Alfonsi P, Adam F, Passard A et al. - Nefopam a nonsedative benzoxazocine analgesic, selectively reduces the shivering threshold in unanesthetized subjects. Anesthesiology, 2004; 100:37-43.

79. Stevens RD, Burri $H$, Tramèr MR - Pharmacologic myocardial protection in patients undergoing noncardiac surgery: a quantitative systematic review. Anesth Analg, 2003;97:623-633.

\section{RESUMEN}

Albergaria VF, Lorentz MN, Lima FAS - Temblores Intra y Postoperatorio: Prevención y Tratamiento Farmacológico.

JUSTIFICATIVA Y OBJETIVOS: Los temblores pueden ocurrir como un efecto adverso de la intervención quirúrgica y anestesia. La incidencia de temblores postoperatorios varía entre 6,3\% y $66 \%$.
Pacientes jóvenes, sexo masculino, uso de agentes anestésicos halogenados y tiempo prolongado de anestesia o procedimiento quirúrgico están relacionados con temblores. Los temblores son involuntarios y se presentan como actividad muscular oscilatoria con finalidad de aumentar la producción de calor. E temblor postoperatorio es una desagradable complicación que está relacionada con el aumento de la morbidez. El temblor aumenta el metabolismo resultando en un aumento de $200 \%$ a $500 \%$ en el consumo de oxígeno.

CONTENIDO: Discute las causas, prevención y tratamiento de los temblores intra y postoperatorio en pacientes adultos y pediátricos sometidos a la intervención quirúrgica bajo anestesia general o del neuroeje.

CONCLUSIONES: Los temblores son, juntamente con náuseas y vómitos, causas de intensa incomodidad en la sala de recuperación pos anestésica, además de potencialmente perjudiciales por generar un aumento de la demanda metabólica. Aunque la presencia de temblores no haya sido directamente relacionada a la morbidez cardiaca, la prevención se ha hecho tema de debate y de varios artículos científicos. La prevención y el tratamiento de temblores deben ser implementados. Pacientes con reserva cardio pulmorar limitada pueden sufrir con la acidosis láctica, falta de saturación venosa mixta e hipoxemia. 